\title{
Fluid Flow and Heat Transfer Characteristics Investigation in the Shell Side of the Branch Baffle Heat Exchanger
}

\author{
K. Wang, J. Q. Liu, Z. C. Liu ${ }^{\dagger}$ W. Chen, X. C. Li and L. Zhang \\ School of Mechanics and Safety Engineering, Zhengzhou University, Zhengzhou 450002, China \\ $\dagger$ Corresponding Author Email: zchliu@zzu.edu.cn
}

(Received December 4, 2020; accepted June 5, 2021)

\begin{abstract}
The branch baffle heat exchanger, being an improved shell-and-tube heat exchanger, for which the flow manner of the shell-side fluid is a mixed flow of oblique flow and local jet. The computational fluid dynamics (CFD) method has been implemented to investigate the fluid pattern and heat transfer performance. The accuracy of the modeling approach has been confirmed by an experimental approach using a Laser Doppler Velocimeter system. Flow field, temperature field, and pressure field are displayed to study the physics behavior of fluid flow and thermal transport. Heat transfer coefficient, pressure drop, and efficiency evaluation criteria are analyzed. In contrast with the shell-and-tube heat exchanger with segmental baffles and shutter baffles, the pressure loss in the proposed heat exchanger with branch baffles has been dramatically improved, accompanied by a slight decrease in heat transfer coefficient under the same volume flow rate. The efficiency evaluation criteria of the heat exchanger with branch baffles are $28 \%-31 \%, 13.2 \%-14.1 \%$ higher than those with segmental baffles and shutter baffles, respectively. Further analysis in accordance with the field synergy principle illustrates that the velocity and pressure gradients of the heat exchanger with branch baffle have finer field coordination. The current heat exchanger structure provides a reference for the future optimization design to reach energy saving and emission reduction.
\end{abstract}

Keywords: Heat exchanger; Branch baffle; CFD; Flow manner; Pressure drop.

\section{NOMENCLATURE}

$A$
$B$
$C$
$c_{\mathrm{p}}$
$d$
$h$
$L_{\mathrm{t}}$
$N_{\mathrm{t}}$
$Q$
$P$
$T_{\text {in }}$
$T_{\mathrm{w}}$
$T_{\text {out }}$
$U$

heat transfer area

baffle spacing

empirical constant

specific heat capacity

outer diameter of tube

shell-side heat transfer coefficient

tube length

number of heat exchange tubes

heat transfer rate

Power consumption

inlet temperature

tube wall temperature

outlet temperature

velocity vector

$\begin{array}{ll}u & \begin{array}{l}\text { flow velocity } \\ V\end{array} \\ W & \begin{array}{l}\text { volume flow rate } \\ \text { grille sheet width }\end{array} \\ \alpha & \begin{array}{l}\text { angle between velocity vector and } \\ \text { temperature gradient }\end{array} \\ \beta & \begin{array}{l}\text { angle between velocity vector and } \\ \text { pressure gradient }\end{array} \\ \Delta p & \begin{array}{l}\text { shell-side pressure drop } \\ \text { turbulence kinetic energy dissipation rate }\end{array} \\ \theta & \begin{array}{l}\text { included angle of grille sheet } \\ \text { dynamic viscosity of viscosity }\end{array} \\ \rho & \text { density }\end{array}$

$u \quad$ flow velocity

$V \quad$ volume flow rate

grille sheet width

angle between velocity vector and temperature gradient

pressure gradient

included angle of grille sheet

density 


\section{INTRODUCTION}

As the core equipment of energy conversion in energy power, especially in the fields of electric power, air conditioning equipment, petrochemical industries, and so on, heat exchangers are of great significance for energy saving of enterprises. As one of the heat exchanger types, the shell-and-tube heat exchanger (STHX) has the advantages of compact structure, low maintenance cost, and simple manufacture, so it is still the most important part of the thermodynamic system (Fan et al. 2012). Supported by the segmental baffle, the shell-side working fluid of the traditional STHX exhibits crossflow characteristics, which has the advantages of simple manufacturing, flexible maintenance, and outstanding heat exchange capability. Nonetheless, the lateral flow also brings the drawbacks of destructive tube bundle vibration, high flow resistance, flow dead zone with fouling, and considerable pump power consumption (Hajabdollahi et al. 2016; Arani and Moradi 2019).

Many shell-side supporting baffles have been proposed and optimized by researchers around the world to improve the flow characteristics of the flowing medium on the shell side in the hope of obtaining better overall heat exchanger performance (Gu et al. 2018a; Wang et al. 2019), such as trefoil-hole baffles (You et al. 2013), rod baffles (Liu et al. 2017; Wang et al. 2017), helical baffles (Lei et al. 2008), unilateral ladder type helical baffles (Chen et al. 2019), ladder-type fold baffles (Wen et al. 2015b), flower baffles (You et al. 2012), louver baffles (Lei et al. 2017), etc. Mellal et al. (2017) numerically researched the influence of baffle arrangement on thermal transport in the shell-and-tube heat exchanger with segmental baffles (SG-STHX) and reported that the baffle orientation of $180^{\circ}$ and baffle spacing of $64 \mathrm{~mm}$ were the optimal design to ensure mixed flow. Wang et al. (2018) expected SG-STHX to have the characteristics of simple fabrication and the helical flow of the STHX with continuous helical baffles and put forward a kind of staggered baffle STHX. Lei et al. (2017) designed two novel STHXs with louver baffles, which are beneficial to avoid the abrupt change in the flow velocity of the shell-side medium. It is disclosed that the pumping power of STHX with louver baffle was below that of SG-STHX at the same heat transfer amount. He and $\mathrm{Li}$ (2018) carried out numerical research on three kinds of double tube-pass STHXs with different baffle configurations, and the CFD simulation findings revealed that the $Q_{\mathrm{H}} / P_{0}$ of flower baffle far exceeded that of segmental baffle. You et al. (2015) employed the circular rods that were arc-cut to support the pipe bundle of STHX. The influences of factors such as baffle spacing, bar diameter, and pipe clamping approach were computationally investigated. Ma et al. (2017) numerically compared the shell-side performance of two longitudinal flow heat exchangers - STHX with trefoil perforated plate and quatrefoil-hole baffle - and found that the improvement of heat transfer efficiency is often accompanied by greater pressure loss. El Makoul et al. (2016) concluded by using the CFD method that the trefoil-hole baffles can improve the shell-side thermal transfer performance, but the performance enhancement comes at the cost of a significant pressure drop. Yang and Liu (2015) developed a new type of STHX with plate baffles and analyzed the temperature field, pressure field, and path lines to prove the advantages of the novel STHX. In response to the problems posed by non-continuous helical baffles in STHXs, Wang et al. (2009) invented a novel continuous helical baffle and conducted a series of studies. Zhang et al. (2013) experimentally compared the features of shell-andtube oil coolers with overlapped helical baffles and segmental baffles. The results indicated that although the former has a lower heat transfer capability, the pressure drop is much smaller than the latter, and the overall performance is enhanced consequently. Wen et al. (2015a) presented an improved structure of ladder-type fold baffle aiming at the triangular leakage zones of the original heat exchangers with helical baffles. The research results showed that the improved baffle can effectively eliminate the axial short circuit flow and improve the heat exchange efficiency. $\mathrm{Gu}$ et al. (2016) used shutter baffles instead of segmental baffles, which synthesized the unique characteristics of transverse flow and longitudinal flow to make the oblique flow of shell-side medium. They found that the comprehensive thermal performance of the STHX was significantly increased, but the heat transfer coefficient was reduced. Based on the cyclical model, Gu et al. (2018b) studied the thermo-fluid characteristics on the shell side of a novel trapezoidal-like tilted baffles heat exchanger.

Kumar et al. (2018) used CFD simulation to analyze the flow manner and heat transfer in a spiral plate heat exchanger, and they reported that the efficiency of the heat exchanger could be promoted by optimizing the fluid flow rate. Mothilal et al. (2018) gained the effect of inlet operating parameters on the thermal transport rate in a cyclone heat exchanger by CFD software FLUENT, and these results were statistically analyzed and experimentally verified. The confirmation test showed that the optimized parameters were able to obtain the maximum heat transfer rate.

Yang et al. (2014) compared the results of rod baffle heat exchanger calculated from four different numerical models with experimental data and reported that the whole model has the highest accuracy in predicting heat transfer, pressure drop, and genuine flow conditions, whereas the whole model requires a large amount of computing resource. As far as research methodology is concerned, experimental results tend to be more convincing and broadly recognized (Yu et al. 2019). However, experiments will consume much time and financial resources. The CFD method with flourishing development is enough to meet the demands of veracity and efficiency (Javadi et al. 2019). Bhutta et al. (2012) summarized the applications of CFD in the field of heat exchangers and indicated that it can effectively predict and 
analyze the performance and behavior of various heat exchangers. Hence, the accuracy and professionalism of the two approaches will be combined in this study.

In summary, the research on improving the heat transfer efficiency of STHXs has been making progress for many years. On this basis, the development of baffles and the improvement of baffles structure to overcome the defects of high flow resistance and high energy consumption of heat exchangers remain a key research direction (Dabrowski et al. 2019) and are completely consistent with the requirements of recent academic progress (Klemes et al. 2020). Depending on the different flow modes of the shell-side operating fluid in the STHX, the flow behavior can be roughly classified into four categories: the crossflow, the longitudinal flow, the helical flow, and oblique flow. Most of the studies are mainly focused on the first three, and the structural optimization design of the oblique flow heat exchanger has been rarely discussed.

The STHX with trefoil-hole baffles is a typical representative of the longitudinal flow heat exchanger, whose shell-side fluid flowing through the trefoil-hole baffles will produce a jet effect. Then, the boundary layer in the heat exchange region is weakened, effectively enhancing heat transfer, but there are some dead zones near the shell wall and baffles (Wang et al. 2019). In the STHX with shutter baffles, the shell-side fluid is divided into multiple streams by the shutter baffles and renders them oblique to scour the tube wall, which can significantly reduce the flow dead zone (Gu et al. 2010). If it is possible to design a supporting structure that could make the shell-side medium flow in a combination of jet and oblique flow to improve the energy efficiency of STHXs. This idea has prompted the authors to conduct the present study. The improved baffle grid is fixedly installed in the shell and is composed of a baffle ring and two grille sheet groups. The two grille sheet groups are staggered in an orthogonal shape and fixedly connected to the inner walls of the baffle ring on both sides. The general structure of the branch baffle shell-and-tube heat exchanger (BB-STHX) is shown in Fig. 1.

Present work discusses and analyzes the medium flow behavior and heat transfer characteristics in the novel STHX. The numerical values are compared with the experimental results of the Laser Doppler Velocimeter (LDV) to verify the reliability of the numerical method (Kumar et al. 2020). Furthermore, the shell-side performance of SGSTHX, shell-and-tube heat exchanger with shutter baffles (SB-STHX), BB-STHX are numerically compared. The mechanism of performance enhancement is explained with the guidance of the field synergy principle. The work in this paper is helpful to design a kind of STHX with high efficiency and energy saving, providing a new scheme and reference for the optimization and upgrading of STHXs and higher energy utilization.

\section{MODEL DESCRIPTION}

\subsection{Physical models of heat exchangers}

The tube supporting structure or baffle is one of the core components of the STHX. By changing the tube supporting structure, the fluid flow manner can be changed, resulting in a difference in the shell-side thermodynamic performance. Because of the wide practicality of the conventional SG-STHX and the oblique flow characteristics of the SB-STHX, these two STHXs were chosen for comparison object to discover the advantages and deficiencies of the BBSTHX.

The branch baffle consists of grille sheets with a certain included angle and baffle rings used to fix grille sheets, as shown in Fig. 2. The sizes of the three types of heat exchangers refer to the national design standard GB/T151. The main geometric parameters of their geometric models are shown in Table 1.

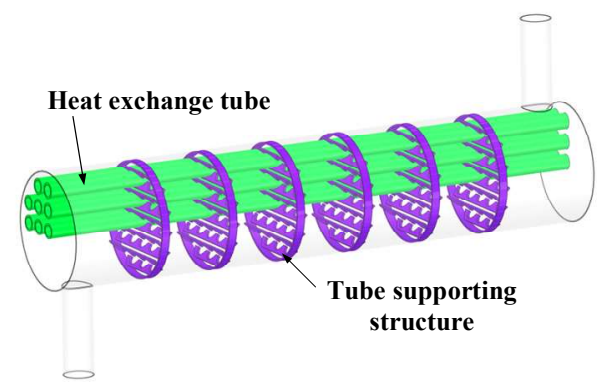

Fig. 1. Geometry model of BB-STHX.

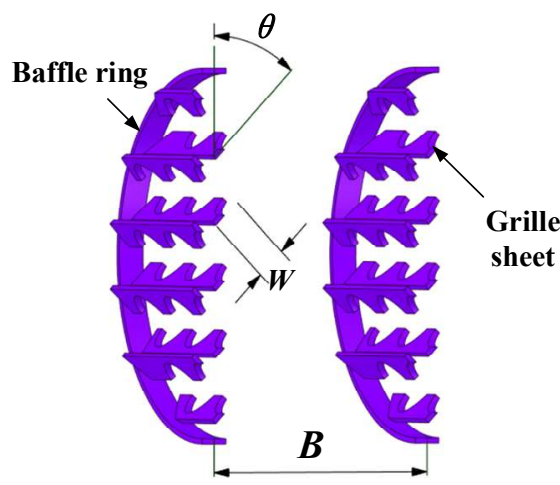

Fig. 2. Structure of tube bundle support of BBSTHX.

\subsection{Numerical simulation}

\subsubsection{Equations}

For stable and incompressible fluids in the computational domain, the governing equations of continuity, momentum, and energy conservation are expressed as follows:

Continuity equation:

$\frac{\partial u_{i}}{\partial x_{i}}=0$ 
K. Wang et al. / JAFM, Vol. 14, No. 6, pp. 1775-1786, 2021.

Table 1 Geometric parameters of STHXs.

\begin{tabular}{|l|c|c|c|}
\hline \multirow{2}{*}{ Parameters } & \multicolumn{3}{|c|}{ Dimensions } \\
\cline { 2 - 4 } & Segmental baffle & Shutter baffle & Branch baffle \\
\hline Inner diameter of shell, mm & 142 & 142 & 142 \\
\hline Tube length, mm & 690 & 690 & 690 \\
\hline Outer diameter of tube, mm & 19 & 19 & 19 \\
\hline Tube pitch, mm & 25 & 25 & 25 \\
\hline Inner diameter of inlet and outlet, mm & 35 & 35 & 35 \\
\hline Baffle cut height, mm & 40.5 & - & - \\
\hline Baffle thickness, mm & 3 & 2 & 2 \\
\hline Baffle pitch, mm & 90 & 90 & 90 \\
\hline Grille sheet width, mm & - & 25 & 12.5 \\
\hline Grille sheet included angle & - & $45^{\circ}$ & $45^{\circ}$ \\
\hline Width of baffle ring, mm & - & 10 & 10 \\
\hline Thickness of baffle ring, mm & - & 2 & 2 \\
\hline Grille sheet pitch, mm & - & 25 & 25 \\
\hline Arranging style for tubes & - & square & square \\
\hline
\end{tabular}

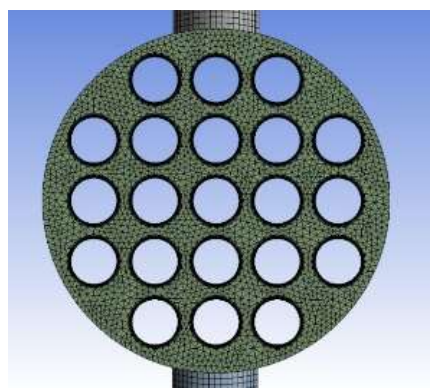

Fig. 3. Sketch of the local grid.

Momentum equation:

$\rho \frac{\partial\left(u_{i} u_{j}\right)}{\partial x_{j}}=-\frac{\partial P}{\partial x_{i}}+\frac{\partial}{\partial x_{j}}\left(\mu \frac{\partial u_{i}}{\partial x_{j}}\right)$

Energy equation:

$\rho \frac{\partial\left(u_{j} T\right)}{\partial x_{j}}=\frac{\partial}{\partial x_{j}}\left(\frac{\lambda}{c_{p}} \frac{\partial T}{\partial x_{j}}\right)$

The standard $\mathrm{k}-\varepsilon$ model is employed to simulate the turbulence. The turbulent kinetic energy part and the turbulent energy dissipation part are listed below, respectively.

$\rho \frac{\partial k}{\partial t}+\rho \frac{\partial\left(k u_{i}\right)}{\partial x_{i}}=\frac{\partial}{\partial x_{j}}\left[\left(\mu+\frac{\mu_{t}}{\sigma_{k}}\right) \frac{\partial k}{\partial x_{j}}\right]+G_{k}-\rho \varepsilon$

$\rho \frac{\partial \varepsilon}{\partial t}+\rho \frac{\partial\left(\varepsilon u_{i}\right)}{\partial x_{i}}=\frac{\partial}{\partial x_{j}}\left[\left(\mu+\frac{\mu_{t}}{\sigma_{\varepsilon}}\right) \frac{\partial \varepsilon}{\partial x_{j}}\right]+\frac{C_{1 \varepsilon} \varepsilon}{k} G_{k}-C_{2 \varepsilon} \rho \frac{\varepsilon^{2}}{k}$

$\mu_{t}=\rho C_{\mu} \frac{k^{2}}{\varepsilon}$

where $C_{\mu}=0.09, C_{1 \varepsilon}=1.44, C_{2 \varepsilon}=1.92, \sigma_{k}=1.0$, $\sigma_{\varepsilon}=1.3$ and $G_{\mathrm{k}}$ is defined as follows:

$G_{k}=\mu_{t}\left(\frac{\partial u_{i}}{\partial x_{j}}+\frac{\partial u_{j}}{\partial x_{i}}\right) \frac{\partial u_{i}}{\partial x_{j}}$

$$
\begin{aligned}
& Q=\frac{\rho V}{3600} \times c_{p} \times\left(T_{\text {out }}-T_{\text {in }}\right) \\
& h=\frac{Q}{A \Delta T_{m}} \\
& A=N_{\mathrm{t}} \pi d L_{\mathrm{t}} \\
& \Delta T_{m}=\frac{\left(T_{\text {out }}-T_{w}\right)-\left(T_{\text {in }}-T_{w}\right)}{\ln \left[\left(T_{w}-T_{\text {in }}\right) /\left(T_{w}-T_{\text {out }}\right)\right]}
\end{aligned}
$$

\subsubsection{Boundary conditions}

The CFD code FLUENT is used to couple turbulence flow and heat transfer. The whole model is established, taking liquid water as the shell-side medium, the inlet adopts the mass flow inlet with an inlet temperature of $293 \mathrm{~K}$, and the outlet is set as the pressure outlet. The heat exchanger tube wall temperature is constant at $343 \mathrm{~K}$, the outer wall surface of the shell side and the grid-baffle are imposed to be adiabatic, and all solid surfaces adopt non-slip boundary conditions. The gravity effect and radiative heat transfer are not taken into consideration and the inlet temperature and flow rate are assumed to be uniform. The standard $\mathrm{k}-\varepsilon$ model is used to predict turbulence ( $\mathrm{Gu}$ et al. 2016; Yu et al. 2020). The SIMPLE algorithm is selected in the coupling method of pressure and velocity.

\subsubsection{Grid generation}

The computational model grid is generated by the commercial software ANSYS Meshing. Because of the complex geometric structure of BB-STHX, the computational domain meshed with tetrahedral and Structured hexahedral grids, which is shown in Fig. 3. The inflation is added near the tube surface. To verify the grid independence, 4 different grid systems were checked when the inlet volume flow was $12.6 \mathrm{~m}^{3} / \mathrm{h}$, the baffle spacing was $90 \mathrm{~mm}$ and the included angle of the grille sheet was $45^{\circ}$. The calculation results and relative deviations of the convective heat transfer coefficient and pressure drop are shown in Table 2. The difference between 
Table 2 Mesh independence verification.

\begin{tabular}{|c|c|c|c|c|}
\hline Number of cells & Heat transfer coefficient, $\mathrm{W} / \mathrm{m}^{2} \cdot \mathrm{K}$ & Deviation, \% & Pressure drop, Pa & Deviation, \% \\
\hline 3253451 & 5400.53 & 1.13 & 17374.16 & -0.58 \\
\hline 3480493 & 5462.01 & 1.35 & 17274.33 & 0.35 \\
\hline 5079359 & 5536.72 & 0.33 & 17334.67 & 0.30 \\
\hline 8288119 & 5555.25 & - & 17387.69 & - \\
\hline
\end{tabular}

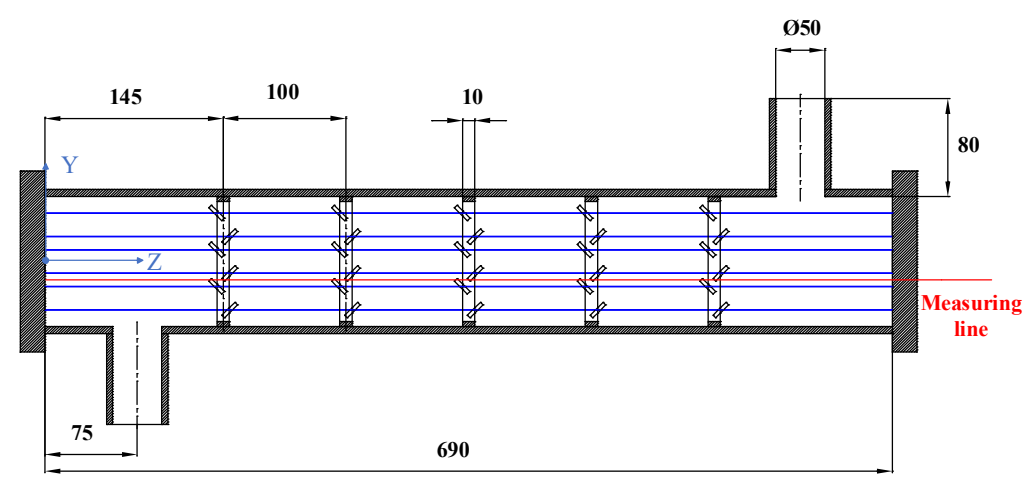

Fig. 4. Axial section of the experimental model.

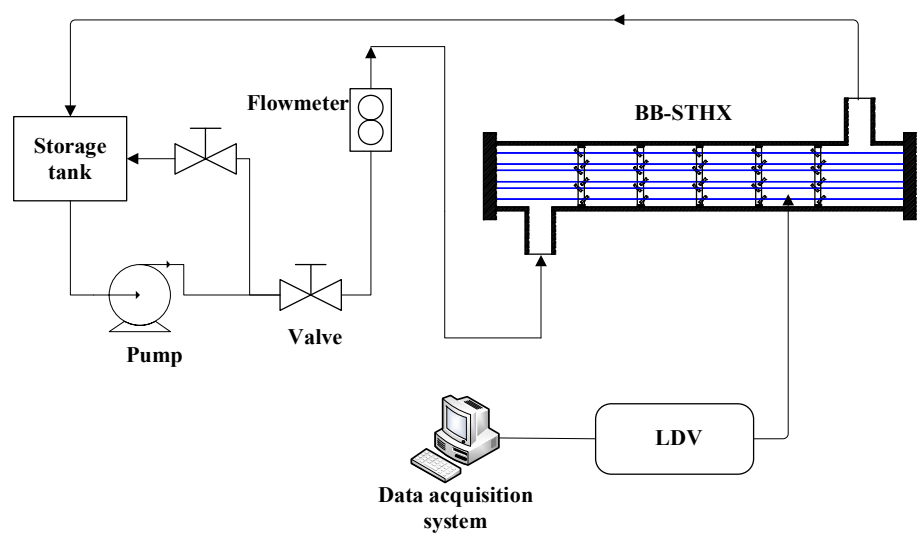

Fig. 5. Flow chart of experimental system.

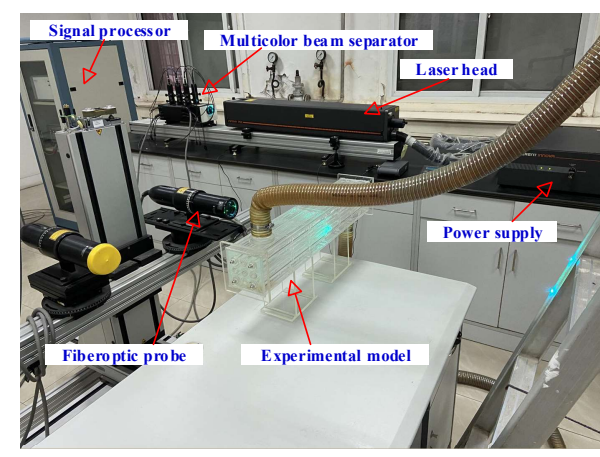

Fig. 6. Photo of experiment.

the heat transfer coefficient and pressure drop with grid numbers of $8.28 \mathrm{M}$ and $5.07 \mathrm{M}$ are both less than $0.33 \%$. Considering the comprehensive calculation capacity, a grid with $5.07 \mathrm{M}$ cells is selected as the calculation model. For all the meshes generated in the computational domain, the maximum skewness does not exceed 0.785 , and the average skewness is between 0.172 and 0.195 , indicating that the mesh quality is relatively good.

\subsection{Experimental verification}

The schematic diagram of the experimental model of BB-STHX is shown in Fig. 4, and the material is plexiglass. Considering the refraction of the laser, the shell of the experimental model is made into a cuboid. The outer tube diameter is $19 \mathrm{~mm}$, and the tube pitch is $30 \mathrm{~mm}$, so that the laser can easily focus on the measuring position. The included angle of grille sheet $\theta$ is $45^{\circ}$, the grille sheet width $W$ is $12.5 \mathrm{~mm}$, the grille sheet spacing $B$ is $30 \mathrm{~mm}$. According to the size of the experimental model, the numerical simulation model is established in equal proportion and calculated. The shell-side flow field of the BB-STHX can be obtained in detail by using the software CFD-POST. The shell-side fluid speed of the experimental model is measured by Laser Doppler Velocimeter (Rothberg et al. 2017), and the results are compared with the results of numerical simulation. Several points on a measuring line parallel to the heat exchanger tube are measured, and 
the velocity components on the y-axis and z-axis of each measuring point are obtained. The measuring line is located at $(0,-15 \mathrm{~mm}, 0) \sim(0,-15 \mathrm{~mm}$, $690 \mathrm{~mm}$ ), as shown in Fig. 4. The flow chart of the experimental system and the experimental photo are shown in Fig. 5 and Fig. 6, respectively.

Figures 7 and 8 show a comparison between the experimental data and the simulation data when the inlet flow is $4.5 \mathrm{~m}^{3} / \mathrm{h}$. As shown, whether it is the shell-side mainstream velocity (z-axis velocity component) or the transverse velocity (y-axis velocity component), the experimental data are in good agreement with the numerical simulation results, the average deviation of mainstream velocity and transverse velocity is about $7.71 \%$ and $9.61 \%$, respectively, and the maximum relative deviation is $19.07 \%$ and $17.81 \%$, respectively. Possible sources of error are as follows: There may be dimensional errors in the processing of the experimental model; There may be positioning errors in the process of selecting measuring points; Calibration of rotor Flowmeter in the hydraulic measuring system. In addition, when the fluid passes through the branch baffles, the velocity is obviously increased, indicating that the jet effect of the baffle is more prominent. And it is evident that the flow velocity has visible periodicity when the fluid crosses the baffle grid.

In order to better illustrate the coincidence degree between the experimental values and the simulation values, two points were selected on the measuring line, and the coordinates are $(0,-15 \mathrm{~mm}, 210 \mathrm{~mm})$ and $(0,-15 \mathrm{~mm}, 450 \mathrm{~mm})$. The mainstream velocities (z-axis flow velocity component) of the two points were measured by taking different volume flow rates of $4.0 \mathrm{~m}^{3} / \mathrm{h}, 4.5 \mathrm{~m}^{3} / \mathrm{h}, 5.0 \mathrm{~m}^{3} / \mathrm{h}, 5.5 \mathrm{~m}^{3} / \mathrm{h}$, and $6 \mathrm{~m}^{3} / \mathrm{h}$, respectively. Table 3 shows the experimental and simulated values and errors of the mainstream velocity at two points under different volume flow rates. The maximum relative error of the two points is less than $9 \%$. The above results show that the established numerical model can be used to determine the characteristics and property of the heat exchanger with high reliability and accuracy.

Table 3 Velocity values of these two measured points

\begin{tabular}{|c|c|c|c|c|c|c|}
\hline \multirow{2}{*}{$\begin{array}{c}\text { Volume } \\
\text { flow rate, } \\
\mathrm{m}^{3} / \mathrm{h}\end{array}$} & \multicolumn{2}{|c|}{$\mathrm{z}=210 \mathrm{~mm}$} & \multirow[b]{2}{*}{ error, $\%$} & \multicolumn{2}{|c|}{$\mathrm{z}=450 \mathrm{~mm}$} & \multirow[b]{2}{*}{ error, $\%$} \\
\hline & $\begin{array}{l}\text { simulation } \\
\text { value, } \mathrm{m} / \mathrm{s}\end{array}$ & $\begin{array}{l}\text { experimental } \\
\text { value, } \mathrm{m} / \mathrm{s}\end{array}$ & & $\begin{array}{l}\text { simulation } \\
\text { value, } \mathrm{m} / \mathrm{s}\end{array}$ & $\begin{array}{l}\text { experimental } \\
\text { value, } \mathrm{m} / \mathrm{s}\end{array}$ & \\
\hline 4.0 & 0.2139 & 0.2317 & 8.33 & 0.0391 & 0.0411 & 5.10 \\
\hline 4.5 & 0.2461 & 0.2542 & 3.27 & 0.0434 & 0.0454 & 4.51 \\
\hline 5.0 & 0.2650 & 0.2754 & 3.93 & 0.0482 & 0.0495 & 2.61 \\
\hline 5.5 & 0.2950 & 0.3079 & 4.37 & 0.0561 & 0.0611 & 8.91 \\
\hline 6.0 & 0.3270 & 0.3464 & 5.93 & 0.0613 & 0.0632 & 3.10 \\
\hline
\end{tabular}

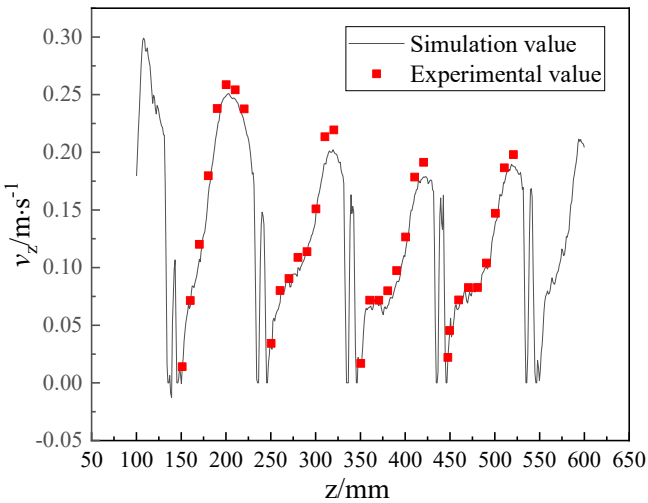

Fig. 7. Comparison of z-axis flow velocity components.

\section{RESUltS AND DisCUSSION}

\subsection{Shell-side fluid flow characteristics}

In the case of shell-side volume flow rate $12.6 \mathrm{~m}^{3} / \mathrm{h}$, Fig. 9 shows the velocity contours and vectors of the three types of heat exchanger models during the full development phase in the longitudinal section. The Reynolds number of the shell-side medium is about 10000 , which is in a state of full turbulence.

As can be seen from Fig. 9(a), the fluid shows an

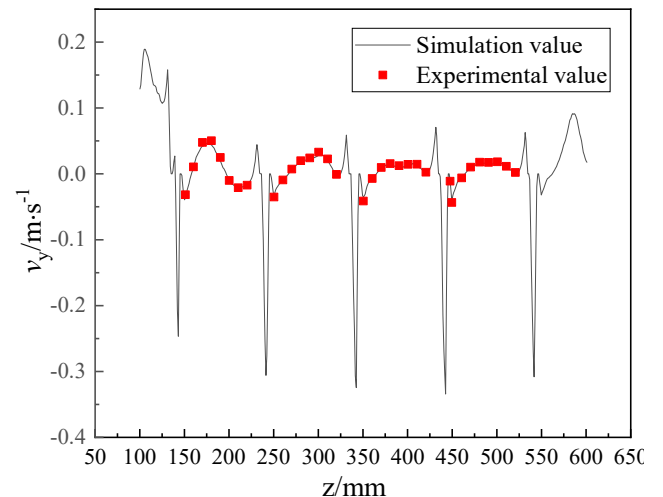

Fig. 8. Comparison of y-axis flow velocity components.

apparent zigzag flow in the fully developed phase of the conventional SG-STHX, and the velocity distribution is extremely uneven. The eddy current retention zone behind the baffle is more likely to scale and corrode the heat exchanger tube. The flow path varies regularly as the fluid contacts the segmented baffle, the velocity at the corner rises sharply, the momentum changes significantly, and the heat transfer area utilization rate is low. The main fluid flows obliquely around the tube at a given angle under the action of the baffles in the fully developed phase of the SB-STHX. And as the guiding influence weakens, the direction is again 


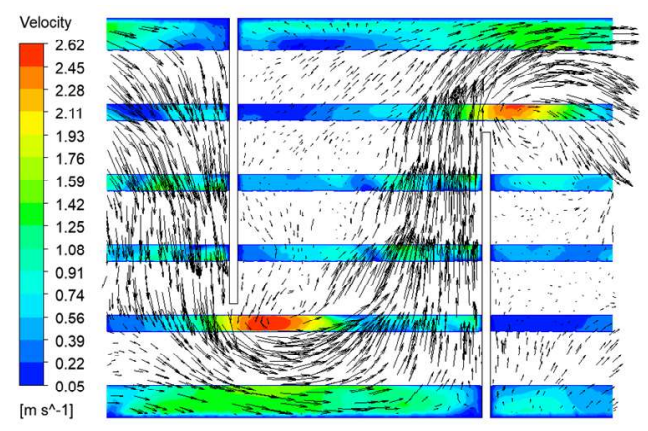

(a) SG-STHX

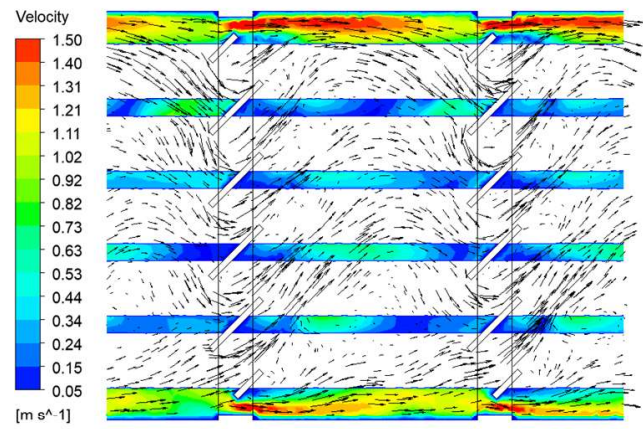

(b) SB-STHX

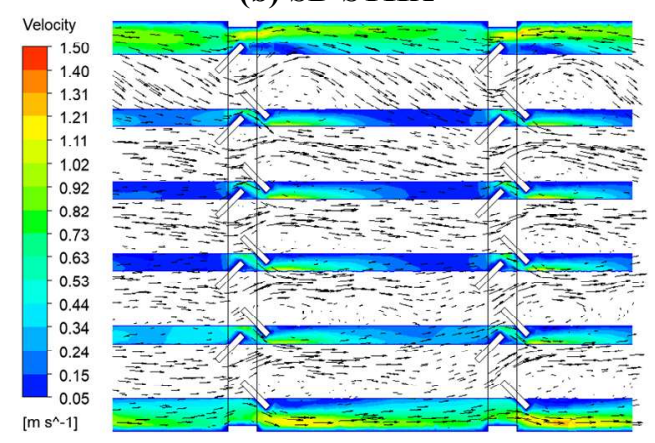

(c) BB-STHX

Fig. 9. Velocity contours superimposed by velocity vectors of three kinds of heat exchangers on longitudinal section.

deflected until the next baffle occurs. As is visible from Fig. 9(b), the shell-side flow induced by the shutter baffle is smoother than the segmental baffle, but the flow velocity is clearly higher on the upper and lower sides than in the center, and to a certain degree there is a circulating flow region behind the baffle. As shown in Fig. 9(c), the medium is no longer a pure oblique flow, but a mixed flow in longitudinal and oblique directions. When the fluid moves through the branch baffle, the fluid flows at a certain angle of included, owing to the special bifurcation arrangement of the branch baffle. And part of the fluid is ejected from the gap at the bifurcation at a higher flow rate, producing a jet effect like that of the STHX with trefoil-hole baffles, which can better scour the pipe surface and diminish the boundary layer thickness. It is not difficult to find that in BB-STHX, the reduction of baffle width and the gap between neighboring baffles will effectively remove the dead zone behind the baffle and develop a smoother shell-side flow field by comparing the velocity field of the three STHXs. Moreover, on the shell side of BB-STHX, the flow velocity is less than that of SB-STHX and SG-STHX, and there is no significant change in the primary flow direction, which can be predicted that the pressure drop in BBSTHX is relatively low as well.

\subsection{Shell-side temperature field and pressure field distribution}

The temperature distributions of three kinds of STHXs in the longitudinal section are seen in Fig. 10 at volume flow rate $V=12.6 \mathrm{~m}^{3} / \mathrm{h}$. The fluid temperature behind the SG-STHX baffle is higher, which is thought to be attributed to the slower speed and increased residence time. The temperature distribution of SB, BB-STHX, nevertheless, is relatively uniform, and the fluid will continuously take away heat from the heat exchanger tube on the shell side, which is advantageous for energy efficiency enhancement. The outlet temperature of BB-STHX is smaller while the inlet temperature is the same. In specific, the outlet temperature of SG, $\mathrm{SB}$, and BB-STHX rises by $18.78 \mathrm{~K}, 14.9 \mathrm{~K}$, and $13.68 \mathrm{~K}$, respectively. Therefore, the heat transfer capability of BB-STHX is inferior to the former two.

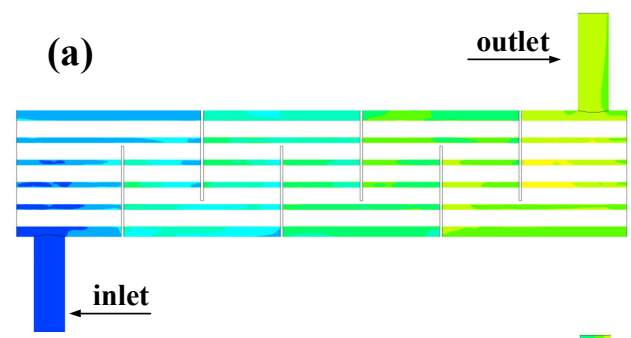

(b)
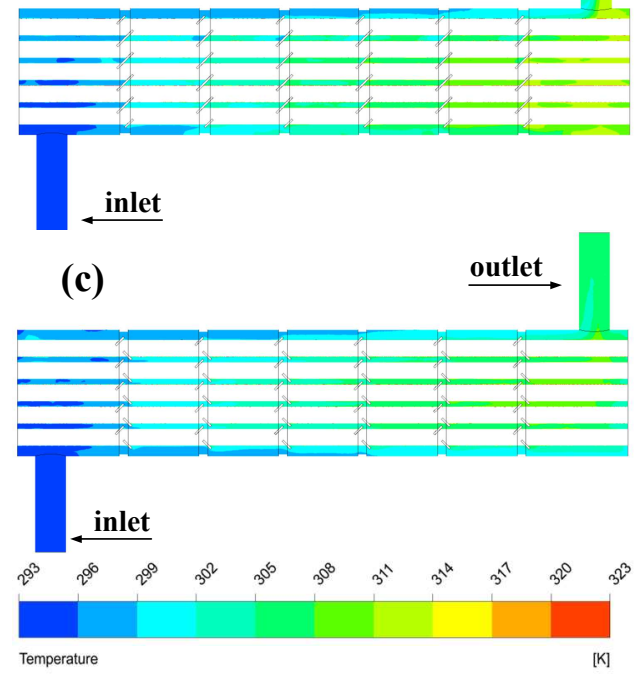

Fig. 10. Shell-side temperature distribution of three kinds of heat exchangers:(a) SG-STHX; (b) SB-STHX; (c) BB-STHX.

The drop in pressure from inlet to outlet of the heat exchanger determines the expense of pumping power to a certain degree and is a parameter that cannot be overlooked (Sarangi et al. 2020). The pressure 
distributions of three kinds of STHXs in the longitudinal section are seen in Fig. 11 at volume flow rate $V=12.6 \mathrm{~m}^{3} / \mathrm{h}$. It can be clearly seen in Fig. 11(a) that there are local abrupt changes in pressure and that the pressure gradient changes obviously, which means that the continuity of fluid flow is weak in the SG-STHX. In Figs. 9(b) and 9(c), the pressure varies uniformly, and the continuity of the fluid is relatively strong. Comparing the inlet and outlet pressures of the three heat exchangers, it can be found that the SG-STHX has the most reduced pressure drop, followed by SB-STHX, and the BBSTHX has the least pressure drop. Quantitatively speaking, the inlet and outlet pressure drops of SG, SB, BB-STHX are $30850 \mathrm{~Pa}, 21393 \mathrm{~Pa}$, and $17274 \mathrm{~Pa}$, respectively. In the BB-STHX, the transition in pressure distribution is smooth, not as abrupt as SGSTHX, which tends to reduce energy consumption and pressure loss.
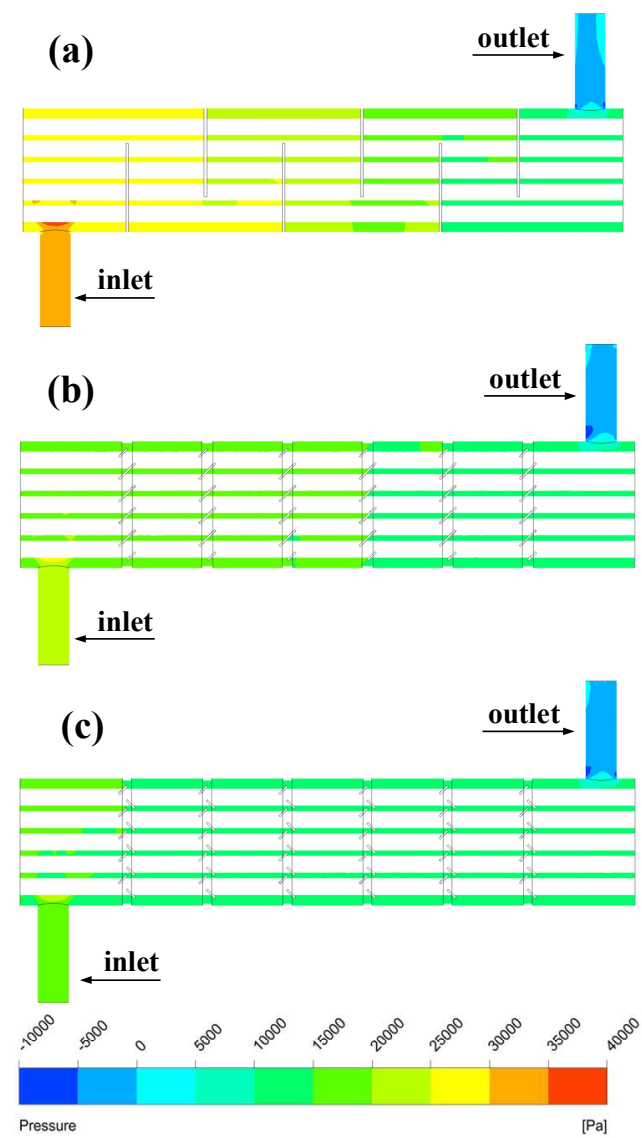

Fig. 11. Shell-side pressure distribution of three kinds of heat exchangers:(a) SG-STHX; (b) SBSTHX; (c) BB-STHX.

\subsection{Performance improvement}

The changes of the shell-side heat transfer coefficient of the three STHXs under different volume flow rates are shown in Fig. 12. For the same volume flow rate, the sequence of heat transfer coefficient from high to low is SG-STHX > SB-STHX > BB-STHX. Where BB-STHX is the lowest, $31.65 \%-32.37 \%$ and $10.76 \%-11.07 \%$ lower than SG-STHX and SBSTHX, respectively. The values of SB-STHX are

\subsection{7\%-24.04\% lower than SG-STHX}

The changes of the shell-side pressure drop of the three STHXs under different volume flow rates are shown in Fig. 13. For the same volume flow rate, the pressure drop in descending order is SG-STHX > SB-STHX > BB-STHX. Where BB-STHX is the smallest, $\quad 43.51 \%-43.90 \%$ and $18.30 \%-19.16 \%$ below SG-STHX and SB-STHX, respectively. The values of SB-STHX are $30.40 \%-30.85 \%$ lower than SG-STHX. For BB-STHX, although the heat transfer capacity is slightly weak, the pressure loss is improved more. The improvement in its comprehensive performance can be predicted.

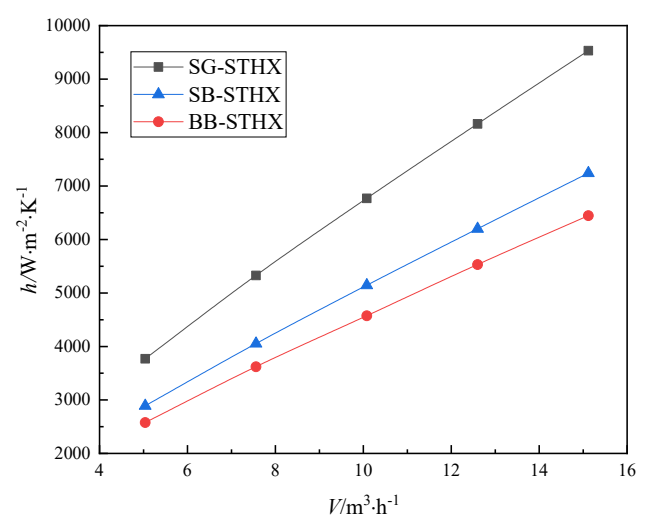

Fig. 12. Variations of shell-side heat transfer coefficient with shell-side volume flow rate.

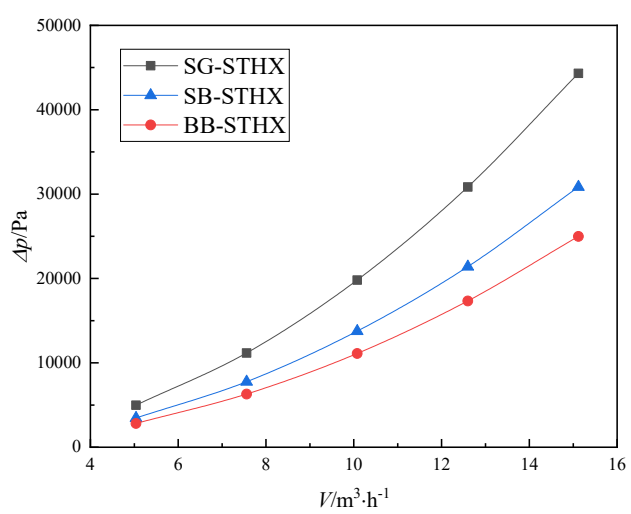

Fig. 13. Variations of shell-side pressure drop with shell-side volume flow rate.

Unilateral evaluation of heat exchanger performance from pressure drop or heat transfer coefficient is onesided, and the efficiency evaluation criterion EEC (Li et al. 2020) is also introduced in this study. EEC evaluates STHX performance based on external input pump power and total heat exchange amount, expressed as:

$$
E E C=\frac{Q / Q_{0}}{P / P_{0}}=\frac{Q / Q_{0}}{(V \Delta p) /\left(V_{0} \Delta p_{0}\right)}
$$

Where $Q$ and $P$ represent the heat transfer amount obtained and external input power, respectively. $\Delta p$ is the shell-side pressure drop. $V$ is the inlet volume flow rate and the subscript " 0 " indicates the comparison object. 


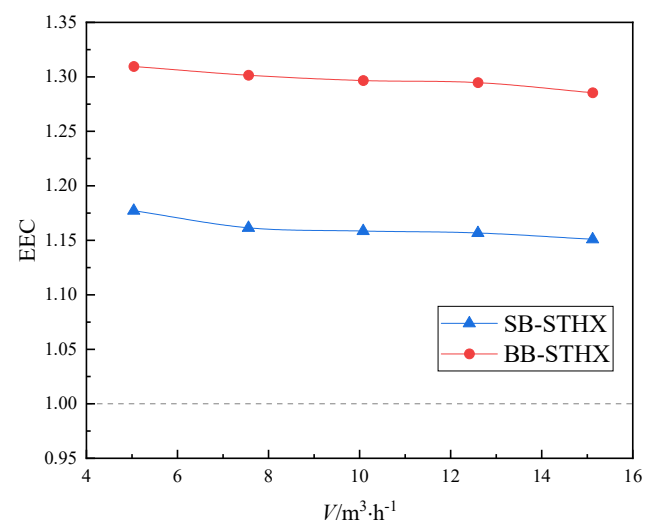

Fig. 14. Variations of EEC value with shell-side volume flow rate.

In this EEC analysis, SG-STHX is chosen as the comparison object. It can be seen from Fig. 14 that the EEC values of both BB-STHX and SB-STHX exceed 1 , and the former is the largest, fluctuating between 1.28-1.31, while the latter is between 1.15 and 1.18. It shows that at a given volume flow rate, the EEC of the BB-STHX higher than that of the SGSTHX by $28 \%-31 \%$, and the EEC of the BB-STHX is always $13.23 \%-14.09 \%$ higher than the EEC of the SB-STHX. In the literature (Gu et al. 2017), the comprehensive performance of SB-STHX was improved by about $7 \%$ by adjusting the assembly mode of the shutter baffle. The difference is that in this paper, by improving the structure of the shutter baffle, a mixed flow form combining oblique flow and local jet can be induced in the main flow area, so the heat transfer efficiency is improved even more, which can prove to be an effective improvement. In actual applications, the STHX pressure drop is often limited, while it is directly related to the pumping power. A heat exchanger with a higher EEC means that the heat transfer rate obtained is greater than the reference object while the pump power conditions are the same. Alternatively, if the design requires the same amount of heat transfer, less external input power is needed. According to the above discussion, it is concluded that BB-STHX exhibits better energy utilization efficiency for the operating conditions interval we have studied.

\subsection{Field synergy principle analysis}

In an effort to explain the performance improvements attributing to the supporting structure, the field synergy principle analysis is applied in this study. The field synergy principle is one of the guidance for the design of heat exchangers, which states that improving the synergy among physical quantities contributes to the efficiency of the heat exchangers (Cao et al. 2019).

The angle between the temperature gradient and the fluid speed vector is referred to as the synergy angle $\alpha$. In the interval $[0,90]$, the smaller the angle $\alpha$, the better the convective heat transfer synergy is implied, and the stronger the heat transfer between the working medium and the wall. The formula for the synergy angle $\alpha$ is expressed as (Liu et al. 2009): $\alpha=\cos ^{-1} \frac{|U \cdot \nabla T|}{|U| \cdot|\nabla T|}$

The angle between the pressure gradient and the fluid speed vector is called the synergy angle $\beta$. In the interval $[90,180]$, a larger synergy angle $\beta$ means a better synergy between the flow and pressure fields, with a lower flow resistance and therefore a less generated pressure drop. The equation for the synergy angle $\beta$ is described as (He et al. 2009):

$\beta=\cos ^{-1} \frac{|U \cdot \nabla p|}{|U| \cdot|\nabla p|}$

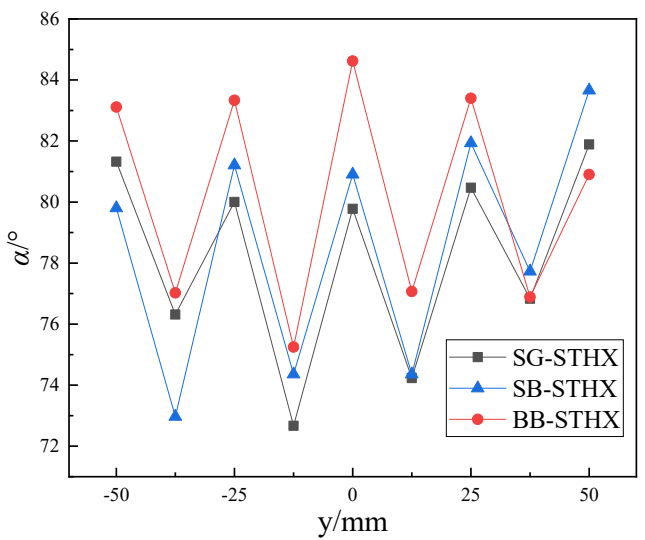

Fig. 15. Average synergy angle $\alpha$ on each slice along the radial direction.

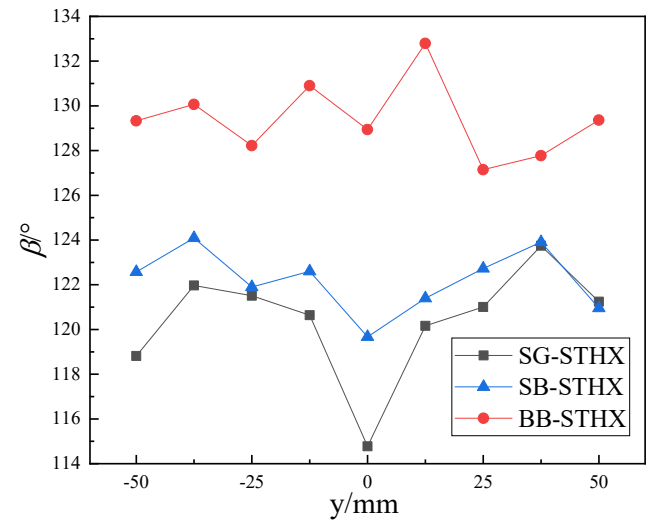

Fig. 16. Average synergy angle $\beta$ on each slice along the radial direction.

Taking the working condition of $V=12.6 \mathrm{~m}^{3} / \mathrm{h}$ as an example, a series of slices of the three STHXs along the radial direction of the shell side were intercepted and the average synergy angle on each slice was calculated separately by coding. The variations of the average synergy angle on each slice along the radial direction are depicted in Figs. 15 and 16, respectively. Herein, the slice with $y=0$ is the central axis slice of the shell. The y-axis positive direction is upward, that is, the direction closer to the outlet nozzle, and the y-axis negative direction is closer to the direction of the inlet nozzle. In overall terms, the mean synergy angle $\alpha$ between velocity and temperature gradient for SG-STHX is smaller than that for SBSTHX on most slices, which is consistent with the 
conclusions reached in the literature (Gu et al. 2016), and furthermore, we additionally find that the mean synergy angle $\beta$ between velocity and pressure gradient for SB-STHX is larger than that for SGSTHX. BB-STHX has larger $\alpha$ and $\beta$ than SB-STHX, which indicates that in BB-STHX, the velocity field has better synergy with the pressure field than in SB and SG-STHX, although the velocity field has slightly less synergy with the temperature field than the other two. The results of the analysis further explain why BB-STHX has a lower heat transfer coefficient and pressure drop. And it agrees well with the shell-side performance results discussed in the previous section.

Moreover, SB-STHX has better synergy between the temperature and velocity fields on the slices close to the inlet nozzle $(y=-50$ and $y=-37.5)$, but poorer synergy on the slices close to the outlet nozzle ( $\mathrm{y}=$ 50 and $y=37.5$ ). BB-STHX has better synergy on the slices close to the outlet nozzle. This may be related to the orientation of the grille sheet. SGSTHX, on the other hand, has the worst synergy between the pressure and velocity fields in the central section. This is due to the shape resistance generated by the segmental baffle.

\section{Conclusion}

In this paper, a novel BB-STHX is introduced. The fluid flow and heat transfer characteristics of three different heat exchangers are analyzed by 3D numerical simulation. An experimental platform was established to verify the accuracy of the whole numerical model. The main findings and results are summarized as follows:

(1). As a result of the bifurcated structure of the branch baffles, the shell-side flow manner in the main flow area is a mixed flow of oblique flow and local jet, thus achieving higher thermal mixing and improved local heat transfer. The primary flow direction hardly changes, and the flow field is uniformly distributed, thus reducing the momentum mutation and pressure loss.

(2). By the comparison of convective heat transfer coefficient $h$, pressure drop $\Delta p$, and efficiency evaluation criterion $E E C$ of the three heat exchangers, although the heat transfer coefficient of BB-STHX slightly decreases, at the same time the pressure loss is improved more significantly. Consequently, its overall performance is $28-31 \%$, 13.2-14.1\% higher than SG, SB-STHX, respectively.

(3) The $\beta$ of BB-STHX is larger than SB, SG-STHX on all radial slices, indicating that it has the best velocity-pressure field synergy. However, the speedtemperature field synergy is weak at most positions, and only the slices near the exit nozzle have better synergy. The mechanism of BB-STHX performance enhancement is explained from the field synergy perspective.

\section{FUTURE SCOPE}

1. This study can be expanded to explore the optimal structural parameters of the branch baffle to improve the heat transfer capacity.

2. This study can additionally be extended to fit empirical correlation equations concerning heat transfer coefficients and pressure drops in heat exchangers.

\section{ACKNOWLEDGEMENTS}

The work is supported by the National Natural Science Foundation Programs of China (No.51776190) and the Independent Innovation Project for Graduate Students of Zhengzhou University.

\section{REFERENCES}

Arani, A. A. A. and R. Moradi (2019). Shell and tube heat exchanger optimization using new baffle and tube configuration. Applied Thermal Engineering 157, 113736.

Bhutta, M. M. A., N. Hayat, M. H. Bashir, A. R. Khan, K. N. Ahmad and S. Khan (2012). CFD applications in various heat exchangers design: A review. Applied Thermal Engineering 32, 112 .

Cao, X., T. Du, Z. Liu, H. Zhai and Z. Duan (2019). Experimental and numerical investigation on heat transfer and fluid flow performance of sextant helical baffle heat exchangers. International Journal of Heat and Mass Transfer 142, 118437.

Chen, J., X. Lu, Q. Wang and M. Zeng (2019). Experimental investigation on thermalhydraulic performance of a novel shell-and-tube heat exchanger with unilateral ladder type helical baffles. Applied Thermal Engineering $161,114099$.

Dabrowski, P., M. Klugmann and D. Mikielewicz (2019). Channel Blockage and Flow Maldistribution during Unsteady Flow in a Model Microchannel Plate heat Exchanger. Journal of Applied Fluid Mechanics 12, 1023 1035.

El Maakoul, A., A. Laknizi, S. Saadeddine, M. El Metoui, A. Zaite, M. Meziane and A. Ben Abdellah (2016). Numerical comparison of shell-side performance for shell and tube heat exchangers with trefoil-hole, helical and segmental baffles. Applied Thermal Engineering 109, 175-185.

Fan, A. W., J. J. Deng, A. Nakayama and W. Liu (2012). Parametric study on turbulent heat transfer and flow characteristics in a circular tube fitted with louvered strip inserts. International Journal of Heat and Mass Transfer 55, 5205-5213.

Gu, X., Q. Dong, M. Liu and Y. Zhou (2010). Numerical Research on Heat Transfer Enhancement of Shutter Baffle Heat Exchanger. Journal of Chemical Engineering of 
K. Wang et al. / JAFM, Vol. 14, No. 6, pp. 1775-1786, 2021.

Chinese Universities 24, 340-345.

Gu, X., B. Liu, Y. Wang and K. Wang (2016). Heat transfer and flow resistance performance of shutter baffle heat exchanger with triangle tube layout in shell side. Advances in Mechanical Engineering 8, 1-8.

Gu, X., Y. Luo, X. Xiong, K. Wang and Y. Wang (2018a). Numerical and experimental investigation of the heat exchanger with trapezoidal baffle. International Journal of Heat and Mass Transfer 127, 598-606.

Gu, X., X. Qin, Y. Wang, D. Zhang and M. Liu (2017). Research on fluid flow and heat transfer characteristics in shell side of inclined shutter baffle heat exchanger. Chemical Industry and Engineering Progress 36, 3584-3589.

Gu, X., Z. Zheng, X. Xiong, T. Wang, Y. Luo and K. Wang (2018b). Characteristics of Fluid Flow and Heat Transfer in the Shell Side of the Trapezoidal-like Tilted Baffles Heat Exchanger. Journal of Thermal Science 27, 602-610.

Hajabdollahi, H., M. Naderi and S. Adimi (2016). A comparative study on the shell and tube and gasket-plate heat exchangers: The economic viewpoint. Applied Thermal Engineering 92, 271-282.

He, L. and P. Li (2018). Numerical investigation on double tube-pass shell-and-tube heat exchangers with different baffle configurations. Applied Thermal Engineering 143, 561-569.

He, Y., Y. Lei, L. Tian, P. Chu and Z. Liu (2009). An Analysis of Three-Field Synergy on Heat Transfer Augmentation with Low Penalty of Pressure Drop. Journal of Engineering Thermophysics 30, 1904-1906.

Javadi, H., S. S. M. Ajarostaghi, M. A. Rosen and M. Pourfallah (2019). Performance of ground heat exchangers: A comprehensive review of recent advances. Energy 178, 207-233.

Klemes, J. J., Q.-W. Wang, P. S. Varbanov, M. Zeng, H. H. Chin, N. S. Lal, N.-Q. Li, B. Wang, X.-C. Wang and T. G. Walmsley (2020). Heat transfer enhancement, intensification and optimisation in heat exchanger network retrofit and operation Renewable \& Sustainable Energy Reviews 120, 109644.

Kumar, A., A. K. Saha, P. K. Panigrahi and A. Karn (2020). Implications of Velocity Ratio on the Characteristics of a Circular Synthetic Jet Flush Mounted on a Torpedo Model in Quiescent and Cross-Flow Conditions. Journal of Applied Fluid Mechanics 13, 1003-1013.

Kumar, K. P. M., V. Vijayan, B. S. Kumar, C. M. Vivek and S. Dinesh (2018). Computational Analysis and Optimization of Spiral Plate Heat Exchanger. Journal of Applied Fluid Mechanics $11,121-128$.

Lei, Y. G., Y. L. He, R. Li and Y. F. Gao (2008). Effects of baffle inclination angle on flow and heat transfer of a heat exchanger with helical baffles. Chemical Engineering and ProcessingProcess Intensification 47, 2336-2345.

Lei, Y., Y. Li, S. Jing, C. Song, Y. Lyu and F. Wang (2017). Design and performance analysis of the novel shell-and-tube heat exchangers with louver baffles. Applied Thermal Engineering $125,870-879$.

Li, N., J. Chen, T. Cheng, J. J. Klemes, P. S. Varbanov, Q. Wang, W. Yang, X. Liu and M. Zeng (2020). Analysing thermal-hydraulic performance and energy efficiency of shell-andtube heat exchangers with longitudinal flow based on experiment and numerical simulation. Energy 202, 117757.

Liu, C., L. Zhang, Y. Xu and Y. Li (2017). CFD study on the radial distribution of coolants in the inlet section of rod-baffle-multi-tubular reactor. Korean Journal of Chemical Engineering 34, 651-663.

Liu, W., Z. Liu, T. Ming and Z. Guo (2009). Physical quantity synergy in laminar flow field and its application in heat transfer enhancement. International Journal of Heat and Mass Transfer 52, 4669-4672.

Ma, L., K. Wang, M. Liu, D. Wang, T. Liu, Y. Wang and Z. Liu (2017). Numerical study on performances of shell-side in trefoil-hole and quatrefoil-hole baffle heat exchangers. Applied Thermal Engineering 123, 1444-1455.

Mellal, M., R. Benzeguir, D. Sahel and H. Ameur (2017). Hydro-thermal shell-side performance evaluation of a shell and tube heat exchanger under different baffle arrangement and orientation. International Journal of Thermal Sciences 121, 138-149.

Mothilal, T., K. Pitchandi, V. Velukumar and K. Parthiban (2018). CFD and Statistical Approach for Optimization of Operating Parameters in a Tangential Cyclone Heat Exchanger. Journal of Applied Fluid Mechanics 11, 459-466.

Rothberg, S. J., M. S. Allen, P. Castellini, D. Di Maio, J. J. J. Dirckx, D. J. Ewins, B. J. Halkon, P. Muyshondt, N. Paone, T. Ryan, H. Steger, E. P. Tomasini, S. Vanlanduit and J. F. Vignola (2017). An international review of laser Doppler vibrometry: Making light work of vibration measurement. Optics and Lasers in Engineering 99, 11-22.

Sarangi, S. K., D. P. Mishra and P. Mishra (2020). Parametric Investigation of Wavy Rectangular Winglets for Heat Transfer Enhancement in a Fin-and-Tube Heat Transfer Surface. Journal of Applied Fluid Mechanics 13, 615-628.

Wang, K., C. Bai, Y. Wang and M. Liu (2019). Flow dead zone analysis and structure optimization for the trefoil-baffle heat exchanger. International Journal of Thermal Sciences 140, 127-134.

Wang, Q., Q. Chen, G. Chen and M. Zeng (2009). Numerical investigation on combined multiple 
K. Wang et al. / JAFM, Vol. 14, No. 6, pp. 1775-1786, 2021.

shell-pass shell-and-tube heat exchanger with continuous helical baffles. International Journal of Heat and Mass Transfer 52, 12141222.

Wang, X., N. Zheng, P. Liu, Z. Liu and W. Liu (2017). Numerical investigation of shell side performance of a double shell side rod baffle heat exchanger. International Journal of Heat and Mass Transfer 108, 2029-2039.

Wang, X., N. Zheng, Z. Liu and W. Liu (2018). Numerical analysis and optimization study on shell-side performances of a shell and tube heat exchanger with staggered baffles. International Journal of Heat and Mass Transfer 124, 247259.

Wen, J., H. Yang, S. Wang, Y. Xue and X. Tong (2015a). Experimental investigation on performance comparison for shell-and-tube heat exchangers with different baffles. International Journal of Heat and Mass Transfer 84, 990-997.

Wen, J., H. Yang, Y. Xue, X. Tong and S. Wang (2015b). Experimental Investigation on Heat Transfer Performance of Heat Exchanger with Ladder-Type Fold Baffles. Journal of Chemical Engineering of Chinese Universities 29, 795801.

Yang, J. and W. Liu (2015). Numerical investigation on a novel shell-and-tube heat exchanger with plate baffles and experimental validation. Energy Conversion and Management 101, 689696.

Yang, J., L. Ma, J. Bock, A. M. Jacobi and W. Liu (2014). A comparison of four numerical modeling approaches for enhanced shell-andtube heat exchangers with experimental validation. Applied Thermal Engineering 65, 369-383.
You, Y., A. Fan, S. Huang and W. Liu (2012). Numerical modeling and experimental validation of heat transfer and flow resistance on the shell side of a shell-and-tube heat exchanger with flower baffles. International Journal of Heat and Mass Transfer 55, 75617569.

You, Y., A. Fan, X. Lai, S. Huang and W. Liu (2013). Experimental and numerical investigations of shell-side thermo-hydraulic performances for shell-and-tube heat exchanger with trefoil-hole baffles. Applied Thermal Engineering 50, 950956.

You, Y., F. Zhang, A. Fan, F. Dai, X. Luo and W. Liu (2015). A numerical study on the turbulent heat transfer enhancement of Rodbaffle heat exchanger with staggered tubes supported by round rods with arc cuts. Applied Thermal Engineering 76, 220-232.

Yu, C., T. Cheng, J. Chen, Z. Ren and M. Zeng (2019). Investigation on thermal-hydraulic performance of parallel-flow shell and tube heat exchanger with a new type of anti-vibration baffle and wire coil using RSM method. International Journal of Thermal Sciences 138 , 351-366.

$\mathrm{Yu}, \mathrm{C}$., H. Zhang, M. Zeng, R. Wang and B. Gao (2020). Numerical study on turbulent heat transfer performance of a new compound parallel flow shell and tube heat exchanger with longitudinal vortex generator. Applied Thermal Engineering 164, 114449.

Zhang, J.-F., S.-L. Guo, Z.-Z. Li, J.-P. Wang, Y.-L. $\mathrm{He}$ and W.-Q. Tao (2013). Experimental performance comparison of shell-and-tube oil coolers with overlapped helical baffles and segmental baffles. Applied Thermal Engineering 58, 336-343. 